\author{
Classification \\ Physics Abstracts \\ $61.25 \mathrm{H}-61.25 \mathrm{E}-64.60 \mathrm{~K}-64.60 \mathrm{C}$
}

\title{
Equilibrium polymerization of liquid sulphur from small angle neutron scattering of sulphur solutions
}

\author{
F. Boué $\left({ }^{2}\right)$, J. P. Ambroise $\left({ }^{2}\right)$, R. Bellissent $\left({ }^{2}\right)$ and P. Pfeuty $\left({ }^{1}\right)$ \\ (1) Laboratoire de Physique des Solides, Université Paris-Sud, 91405 Orsay, France and \\ Laboratoire Léon Brillouin (CEA-CNRS), CE-Saclay, 91191 Gif-sur-Yvette Cedex, France \\ ( ${ }^{2}$ ) Laboratoire Léon Brillouin (CEA-CNRS), CE-Saclay, 91191 Gif-sur-Yvette Cedex, France
}

(Received 26 February 1992, accepted in final form 30 March 1992)

\begin{abstract}
The reversible singular anomaly which shows up in liquid sulphur at $159{ }^{\circ} \mathrm{C}$ and is also present in sulphur solutions has been clearly detected for the first time by small angle neutron scattering in solutions of sulphur with deuterated naphtalene and of sulphur with deuterated biphenyl. The observed sudden rise of the small $\mathrm{q}$ limit of the scattering intensity is interpreted as the signature of an equilibrium polymerization transition with formation of long sulphur chains. Experimental measurements are in qualitative agreement with theoretical predictions based on the mean field approximation of a lattice model.
\end{abstract}

\section{Introduction.}

Sulphur presents a large polymorphism in both solid liquid and vapor phases [1]. Liquid sulphur is certainly with helium one of the most complex monoatomic liquids. It has received scientific attention for almost two centuries and is still not well understood. Solid sulphur melts around $120^{\circ} \mathrm{C}$ at atmospheric pressure to give a pale yellow rather fluid liquid (with a viscosity about ten times the viscosity of water). Around $T_{\mathrm{p}}^{*}=159{ }^{\circ} \mathrm{C}$ it presents a sudden reversible change. Its viscosity changes by four orders of magnitude in a small temperature interval [2] and its color goes from yellow to a luminous orange red to a deep dark red and finally to brown. It also presents a rather sharp $\lambda$ shape specific heat peak and all its physical properties (density, sound velocity, conductivity...) are anomalous around $T_{\mathrm{p}}^{*}[1]$. The « new » liquid above $T_{\mathrm{p}}^{*}$ can be quenched to give a plastic sulphur with rubber like properties.

After a long gestation period beginning with the work of Berthelot in 1857 [3] and leading to a two fluid picture $\left(S_{\lambda}\right.$ and $\left.S_{\mu}\right)$ the « equilibrium polymerization model » emerged around 1960 [4] : liquid sulphur below $T_{\mathrm{p}}^{*}=159^{\circ} \mathrm{C}$ consists mainly of crown shape $S_{8}$ rings (Fig. 1). At $T_{\mathrm{p}}^{*}$ some rings break to build a few very long chains and the liquid above $T_{p}$ is a mixture of $S_{8}$ rings $\left(S_{\lambda}\right)$ and of long chains of all sizes in dynamic equilibrium $\left(\mathrm{S}_{\mu}\right)$. The polymerization transition at $T_{\mathrm{p}}^{*}$ is driven by entropic forces and recently the «equilibrium polymerization model » has been refined to take into account the chain configurational fluctuations [5]. The polymerization transition can then be described by the 


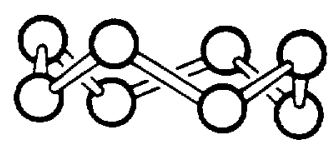

$$
V_{M}=236 \AA^{3} S_{8}(256)
$$

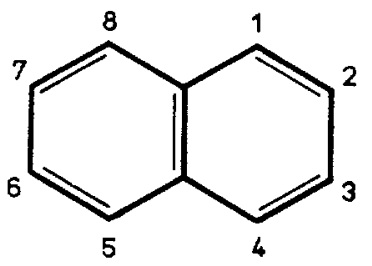

$$
V_{M}=221 \AA^{3} C_{10} D_{8}(136)
$$

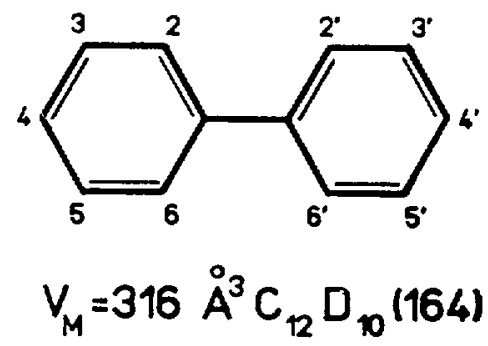

Fig. 1. - Structure of the molecular species present in the different solutions : sulphur $S_{8}$ rings, naphtalene $d C_{10} D_{8}$, biphenyl $d_{C_{12}} \mathrm{D}_{10}$. We have indicated the molecular weight and the molecular volume at $120^{\circ} \mathrm{C}$.

$n \rightarrow 0$ limit of the $O(n)$ model $[5,6]$ and is analogous to a second order phase transition with critical effects. The actual view is that the transition at $T_{\mathrm{p}}^{*}$ is a second order polymerization transition slightly rounded by the presence of an effective very small « field " coupled to the «order parameter». Close to the transition critical fluctuations are associated with the conformational fluctuations of the very long chains and scaling properties of polymers are recovered [7].

Recently the transition was examined at the scale of a few Angströms from large angle neutron scattering study [8]. The opening of the $S_{8}$ rings to build chains is reflected by a change in the pair correlation function $g(r)$ in the range $4 \AA<r<5 \AA$. At a larger scale the long chains should be "seen " through small angle neutron scattering. Unfortunately, the only contrast comes from the difference in specific volume for sulphur in chains and sulphur in $\mathrm{S}_{8}$ rings. This difference is much too small as deduced from the study of sulphur density [9]. On the other hand, by considering sulphur solutions we can get a good contrast with respect to the solvent. These solutions can be prepared with a large variety of solvents and have been studied experimentally $[10,11]$ and theoretically $[12,13]$. The polymerization transition still 
exists in solutions and can be detected from viscosity measurements [10]. Moreover the polymerization transition may induce a phase separation at a lower critical solution point (LCSP) which is closely related to a tricritical point [13]. We shall present here small angle neutron scattering results from solutions with several concentrations of sulphur with deuterated naphtalene and of sulphur with deuterated biphenyl where in the second case a LCSP is present [11]. The growing of sulphur chains cannot be seen directly. Through neutron scattering, we have access to the concentration correlation function $S(q)$ and we can thus see only the sulphur atoms with respect to the solvent molecules without distinguishing between atoms in $S_{8}$ rings and atoms in chains. However the polymerization will induce strong long range concentration fluctuations which we have observed at the transition. The scattering intensity $I(q)$ which is flat below $T_{\mathrm{p}}^{*}$ is becoming peaked at small $q$ above $T_{\mathrm{p}}^{*}$. The limit $I(q \rightarrow 0)$ which represents the concentration susceptibility increases rapidly in the vicinity of $T_{\mathrm{p}}^{*}$. A similar behavior has been observed in $\mathrm{He}_{3}-\mathrm{He}_{4}$ solutions $[14,15]$. The article is structured as follows: in section 2 , we describe the experimental methods (sample preparation, data acquisition and treatment) ; in section 3, the results for the scattered intensity $I(q)$ are discussed. In section 4 theoretical predictions are developed for the correlation function $S(q)$ from a lattice model of sulphur solutions [13] and compared with the experiments. Finally a summary is presented in section 5 .

\section{Experimental methods.}

2.1 SAMPle PREPARATION. - Mixtures of sulphur and naphtalene d (biphenyl d) (Fig. 1) both in the solid state at room temperature are prepared with $X_{\mathrm{s}}$ being the molar fraction of sulphur. An inox (Z2CN-18-10) cell (Fig. 2), $30 \mathrm{~mm}$ long and $29 \mathrm{~mm}$ in diameter is filled with the mixture on a heating plate so that the solution is liquid with a maximum volume of $2 \mathrm{~cm}^{3}$. The cell is then cooled and closed with a quartz window and a teflon seal (Fig. 2). All these operations are made in a dry box under nitrogen atmosphere. Sulphur powder has been purchased from Aldrich (99.999), naphtalene d from CEA-Saclay and biphenyl d from Aldrich. The cell is then placed in the middle of an inox (Z3 CN17-12) cylindrical oven $148 \mathrm{~mm}$ in length and $30 \mathrm{~mm}$ in diameter (with a heating length of $138 \mathrm{~mm}$ ). The oven is placed inside a box with quartz windows and filled with low pressure helium. Type $\mathrm{K}$ (chromel alumel) thermocouples are used for regulation and temperature measurements.

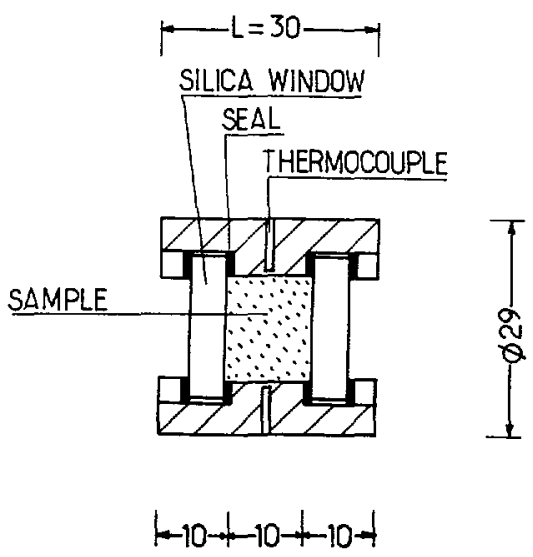

CELL

Fig. 2. - Transverse view of the cylindrical cell. 
2.2 DATA ACQUiSITION AND TREATMENT. - The oven is placed at the " sample position » on the small angle spectrometer PACE in LLB at Saclay at a distance $D=323 \mathrm{~cm}$ of a multidetector made of 30 concentric rings of radius $r_{i}=(3+i) \mathrm{cm}, i=0,29$. The incident neutron beam has a selected wavelength $\lambda=6.57 \AA$ and is collimated through boron nitride diaphragms $10 \mathrm{~mm}$ in diameter placed at both faces of the cell. The scattered intensity $I(q)$ is directly recorded on each of the 30 rings of the multidetector so that the momentum $q$ takes the 30 discrete values $q_{t}=\frac{4 \pi}{\lambda} \sin \left[\frac{\operatorname{arctg}}{2}\left(\frac{r_{i}}{D}\right)\right]$ with $i$ varying from 0 to 29 and thus ranges from $8.88 \times 10^{-3} \AA^{-1}$ to $9.44 \times 10^{-2} \AA^{-1} I_{\mathrm{e}}(q), I_{\mathrm{s}}(q), I_{\mathrm{D}}(q)$ correspond respectively to the scattering intensity, for the empty cell, for the cell filled with the sample and for the cell filled with $\mathrm{D}_{2} \mathrm{O}$ for taking into account the detectors efficiency. The corresponding transmission coefficients $T_{\mathrm{e}}, T_{\mathrm{s}}, T_{\mathrm{D}}$ have been measured. By comparison with scattering by a $1 \mathrm{~mm}$ thick $\mathrm{H}_{2} \mathrm{O}$ cell the scattering intensity for $\mathrm{D}_{2} \mathrm{O}$ (with $1 \mathrm{~cm}$ thick sample) is measured to be $0.04 \mathrm{~cm}^{-1}$ The measured scattering intensity of the sample evaluated in $\mathrm{cm}^{-1}$ is given by

$$
I(q)=0.04 \mathrm{~cm}^{-1} \times \frac{\frac{I_{\mathrm{s}}(q)}{T_{\mathrm{s}}}-\frac{I_{\mathrm{e}}(q)}{T_{\mathrm{e}}}}{\frac{I_{\mathrm{D}}(q)}{T_{\mathrm{D}}}-\frac{I_{\mathrm{e}}(q)}{T_{\mathrm{e}}}}
$$

$I(q)$ has been obtained as a function of temperature in the range $160^{\circ} \mathrm{C}<T<260^{\circ} \mathrm{C}$ for pure sulphur, pure naphtalene $d$, for mixtures of sulphur and naphtalene $d\left(X_{s}=0.9\right.$, $0.8,0.7,0.6,0.55)$ and for mixtures of sulphur and biphenyl $\mathrm{d}\left(X_{\mathrm{s}}=0.82\right.$ and $\left.X_{\mathrm{s}}=0.75\right)$.

\section{Results and discussion.}

3.1 EXPERIMENTAL RESULTS. - In figure 3, $I(q)$ in $\mathrm{cm}^{-1}$ is represented as a function of $q$ in $\AA^{-1}$ for sulphur + naphtalene $\mathrm{d}$ with $X_{\mathrm{s}}=0.6$ and four different temperatures between $220^{\circ} \mathrm{C}$ and $250^{\circ} \mathrm{C}$. In figure 4 similar results are shown for sulphur and biphenyl d with $X_{\mathrm{s}}=0.82$ and four different temperatures between $180^{\circ} \mathrm{C}$ and $235^{\circ} \mathrm{C} . I(q)$ has also been measured on D11 at ILL Grenoble for sulphur-naphtalene d solutions for $q$ values

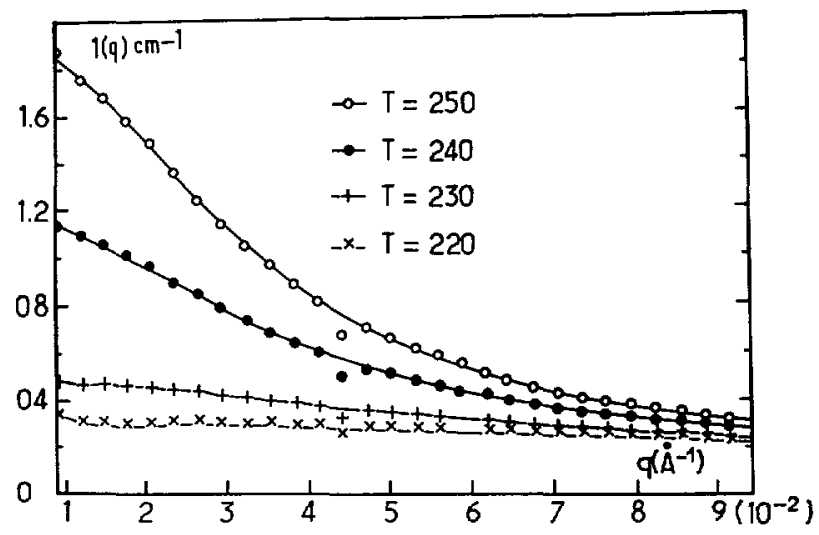

Fig. 3. $-I(q)$ in $\mathrm{cm}^{-1}$ as a function of $q \mathrm{~A}^{-1}$ for sulphur + naphtalene $\mathrm{d}$ with $X_{\mathrm{s}}=0.60$ for four different temperatures $T=220^{\circ} \mathrm{C}, 230{ }^{\circ} \mathrm{C}, 240^{\circ} \mathrm{C}$ and $250^{\circ} \mathrm{C}$ (the systematic deviation of the data points at $q=0.044 \AA^{-1}$ is due to a failure of the detector ring $n^{r} 13$ ). 


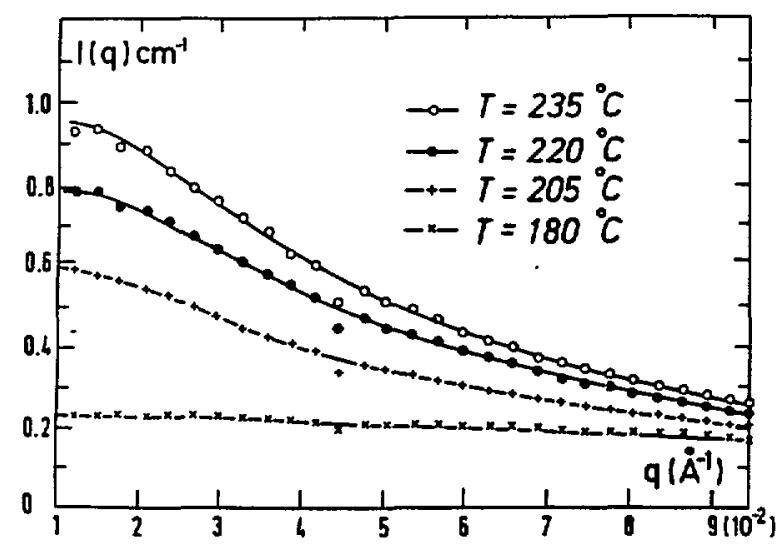

Fig. 4. $-I(q)$ in $\mathrm{cm}^{-1}$ as a function of $q \mathrm{~A}^{-1}$ for sulphur + biphenyl d with $X_{\mathrm{s}}=0.82$ for four different temperatures $T=180^{\circ} \mathrm{C}, 205^{\circ} \mathrm{C}, 220^{\circ} \mathrm{C}$ and $235^{\circ} \mathrm{C}$ (the systematic deviation of the data points at $q=0.044 \AA^{-1}$ is due to a failure of the detector ring $n^{r} 13$ ).

between $0.6 \times 10^{-2} \AA^{-1}$ and $2.4 \times 10^{-2} \AA^{-1}$. There is still a curvature in $I(q)$ at small $q$ so that it is difficult to extrapolate towards $q \rightarrow 0$, because even a Zimm representation $l^{-1}=f\left(q^{2}\right)$ is not linear. This non linearity is mainly due to the fact that $I(q)$ represents the concentration fluctuations for both sulphur in chains and sulphur in $S_{8}$ rings. We thus decided to show in figures 5 and 6 a plot of $I\left(q_{\mathrm{m}}\right)$ with $q_{\mathrm{m}}=0.9 \times 10^{-2} \AA^{-1}$ (that we still call $I(0))$, as function of temperature respectively for sulphur and naphtalene $\mathrm{d}\left(X_{\mathrm{s}}=0.7\right.$ and

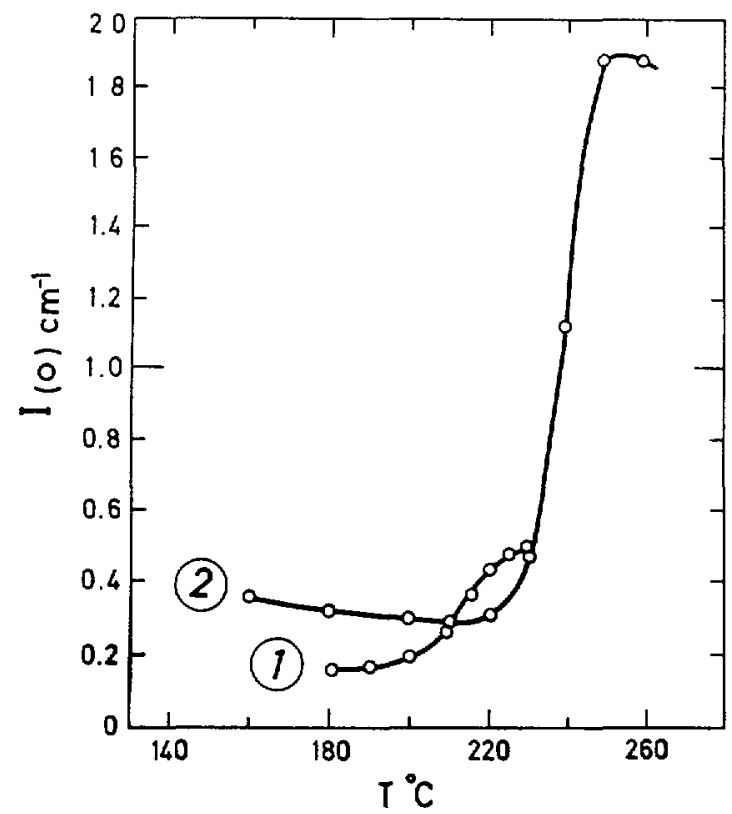

Fig. 5. - Scattering intensity at $q=0.888 \times 10^{-2} \AA^{-1}$ (called $I(0)$ ) in $\mathrm{cm}^{-1}$ as function of temperature for sulphur + naphtalene d. Data points on line 1 correspond to $X_{s}=0.7$ and on line 2 to $X_{s}=0.6$. The drawn lines are only guides to the eye. 


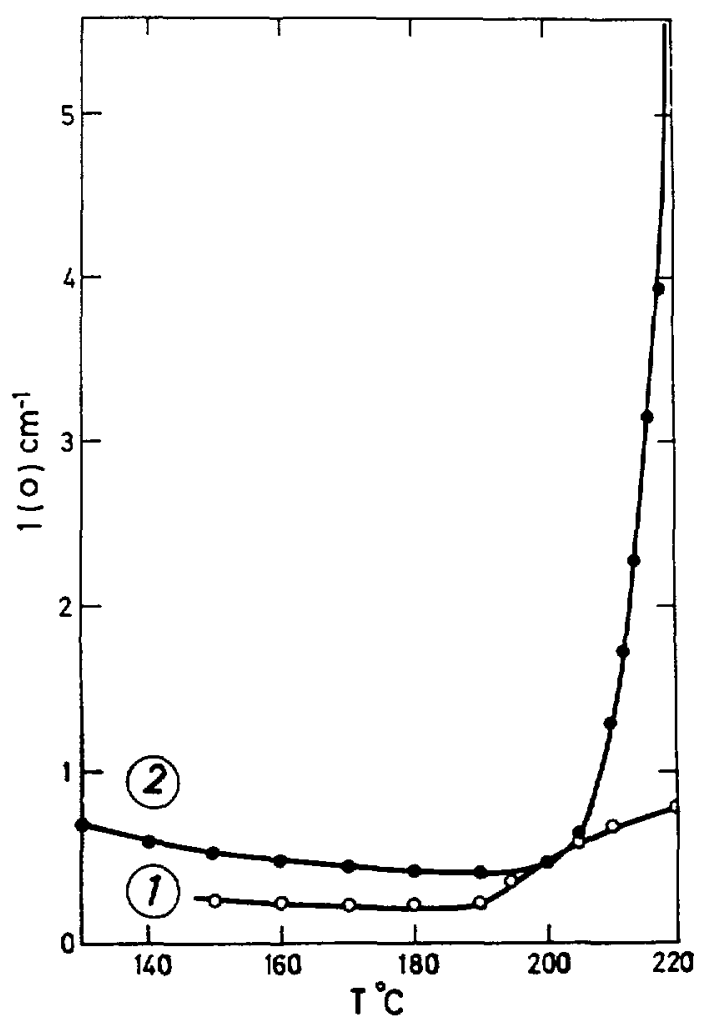

Fig. 6. - Scattering intensity at $q=0.888 \times 10^{-2} \AA^{-1}$ (called $I(0)$ ) in $\mathrm{cm}^{-1}$ as function of temperature for sulphur + biphenyl d. Line 1 corresponds to $X_{s}=0.82$ and on line 2 to $X_{s}=0.75$. The drawn lines are only guides to the eye.

$0.6)$ and for sulphur and biphenyl $\mathrm{d}\left(X_{\mathrm{s}}=0.82\right.$ and 0.75$)$. Below a transition temperature $T_{\mathrm{p}}\left(X_{\mathrm{s}}\right)$ which depends mainly on the concentration $X_{s}$, the spectrum $I(q)$ is almost flat as expected for a binary mixture away from the phase separation. When $T$ approaches $T_{\mathrm{p}}\left(X_{\mathrm{s}}\right)$ the spectrum gets peaked at small $q$ and $I(0)$ defined above and representing the concentration susceptibility is increasing rapidly as shown in figures 5 and 6 with a levelling off at higher temperature : $I(0)$ presents a rounded step like shape reminiscent of the behavior of the concentration susceptibility in $\mathrm{He}_{3}-\mathrm{He}_{4}$ solution close to the superfluid transition [14]. The slow decrease of $I(0)$ before the rapid rise at $T_{\mathrm{p}}$ is due to the presence of an ordinary upper critical point at lower temperature. The height of the step of $I(0)$ around $T_{p}\left(X_{s}\right)$ depends much on $X_{s}$. It increases when $X_{s}$ gets smaller and vanishes to zero when $X_{s}$ goes to one (pure sulphur).

3.2 CONTRAST AND RELATION BETWEEN $I(q)$ AND $S(q)$. - The scattered intensity $I(q)$ is the sum of four main contributions

$$
I(q)=I_{0}+I_{1}+I_{2}+I_{3}
$$

$I_{0}(q)$ is the coherent scattering proportional to the concentration correlation function $S(q)$. Following Bhatia and Thornton [16]

$$
I_{0}=A S(q)=\frac{1}{V}\left(b_{2} \frac{V_{1}}{V}-b_{1} \frac{V_{2}}{V}\right)^{2} S(q)
$$


where $b_{1}$ and $b_{2}$ are respectively the coherent scattering length of the molecule $S_{8}$ and of the solvent molecule and $V_{1}, V_{2}$ their respective partial volumes. $V$ is the mean molecular volume $\left(V=X_{\mathrm{s}} V_{1}+\left(1-X_{\mathrm{s}}\right) V_{2}\right)$. The contrast $A$ is mainly due to the difference $b_{2}-b_{1}$ and depends slightly on $X_{s}$ as shown in table I. The second contribution is the incoherent scattering which is $q$ independent. Natural sulphur has no incoherent scattering, so that $I_{1}$ is only due to the solvent

$$
I_{1}=\left(1-X_{s}\right) \frac{\sigma_{2}}{4 \pi}
$$

Table I. - Various contributions and contrast as function of concentration $X_{s}$ (calculated). A. Sulphur + naphtalene d.

\begin{tabular}{|l|l|l|r|}
\hline$X_{\mathrm{s}}$ & $A\left(\mathrm{~cm}^{-1}\right)$ & \multicolumn{1}{|c|}{$I_{1}\left(\mathrm{~cm}^{-1}\right)$} & \multicolumn{1}{|c|}{$I_{2}\left(\mathrm{~cm}^{-1}\right)$} \\
\hline 1 & 0.41 & 0 & $1.6 \times 10^{-4}$ \\
0.9 & 0.42 & $0.5 \times 10^{-3}$ & $4 \times 10^{-4}$ \\
0.8 & 0.425 & $1.0 \times 10^{-3}$ & $7 \times 10^{-4}$ \\
0.7 & 0.435 & $1.6 \times 10^{-3}$ & $11 \times 10^{-4}$ \\
0.6 & 0.443 & $2.2 \times 10^{-3}$ & $16 \times 10^{-4}$ \\
0.55 & 0.447 & $2.5 \times 10^{-3}$ & $20 \times 10^{-4}$ \\
0 & 0.5 & $5.7 \times 10^{-3}$ & $83 \times 10^{-4}$ \\
\hline
\end{tabular}

B. Sulphur + biphenyl d.

\begin{tabular}{|l|l|l|r|}
\hline$X_{\mathrm{s}}$ & $A\left(\mathrm{~cm}^{-1}\right)$ & \multicolumn{1}{|c|}{$I_{1}\left(\mathrm{~cm}^{-1}\right)$} & \multicolumn{1}{|c|}{$I_{2}\left(\mathrm{~cm}^{-1}\right)$} \\
\hline 1 & 0.57 & 0 & $1.6 \times 10^{-4}$ \\
0.9 & 0.51 & $0.6 \times 10^{-3}$ & $4 \times 10^{-4}$ \\
0.82 & 0.48 & $1.1 \times 10^{-3}$ & $7 \times 10^{-4}$ \\
0.8 & 0.47 & $1.3 \times 10^{-3}$ & $8 \times 10^{-4}$ \\
0.75 & 0.45 & $1.5 \times 10^{-3}$ & $9.5 \times 10^{-4}$ \\
0.7 & 0.43 & $1.8 \times 10^{-3}$ & $12 \times 10^{-4}$ \\
0.5 & 0.36 & $2.9 \times 10^{-3}$ & $23 \times 10^{-4}$ \\
0 & 0.24 & $5 \times 10^{-3}$ & $63 \times 10^{-4}$ \\
\hline
\end{tabular}

The value of $\beta_{\mathrm{T}}$ used is taken equal to $X_{\mathrm{s}} \beta_{\mathrm{T} 1}+\left(1-X_{\mathrm{S}}\right) \beta_{\mathrm{T} 2}$ with $\beta_{\mathrm{T} 1}$ (sulphur) $=$ $3.2 \times 10^{-10} \mathrm{~N}^{-1} \mathrm{~m}^{2} ; \beta_{\mathrm{T} 2}$ (Napht. or Biphenyl $)=4.6 \times 10^{-10} \mathrm{~N}^{-1} \mathrm{~m}^{2}$.

where $\sigma_{2}$ is the incoherent scattering cross section of the solvent molecule. The third contribution $I_{2}$ is the coherent scattering due to density fluctuations which for small $q$ is proportional to the compressibility $\beta_{\mathrm{T}}$

$$
I_{2}=\frac{\left(X_{\mathrm{s}} b_{1}+\left(1-X_{s}\right) b_{2}\right)^{2}}{V^{2}} k T \beta_{\mathrm{T}}
$$

$I_{2}$ depends slightly on $X_{\mathrm{s}}$ and on temperature. Estimates of $I_{1}$ and $I_{2}$ shown in table $\mathrm{I}$ are small of the order of $10^{-3} \mathrm{~cm}^{-1}$ for solutions with sulphur concentrations around $X_{\mathrm{s}}=0.7$. 
The last contribution $I_{3}$ is due to inelastic corrections and to multiple scattering which is non negligible for samples $10 \mathrm{~mm}$ thick with transmission coefficients of 0.6 . This correction is mainly linked to $I_{1}+I_{2}$ and not to $I_{0}$ which is decaying rapidly at large angle. Thus $I_{3}$ can be estimated from a study of the pure solvent $\left(X_{\mathrm{s}}=0\right)$. For pure naphtalene d we got a $q$ independent scattering intensity of $0.037 \mathrm{~cm}^{-1}$ at $120^{\circ} \mathrm{C}$ and $0.050 \mathrm{~cm}^{-1}$ at $200{ }^{\circ} \mathrm{C}$. This gives, from equation (3.1) and calculated values of $I_{1}$ and $I_{2}$ given in table $I$, an estimate for $I_{3} \sim 0.02 \mathrm{~cm}^{-1}$ (we got the same estimate by measuring the scattering intensity of $\mathrm{D}_{2} \mathrm{O}$ in the same cell). We finally relate $S(q)$ to the measured $I(q)$ through the relation

$$
S(q)=\frac{1}{A}\left[I(q)-I_{1}-I_{2}-I_{3}\right]
$$

where $I_{1}$ and $I_{2}$ are given in table I and $I_{3} \sim 0.02\left(1-X_{\mathrm{s}}\right) \mathrm{cm}^{-1}$ The volume $V$ used in the various estimates is certainly underestimate and should be determined from density measurements. For $X_{\mathrm{s}}=0.7$ the contribution $I_{1}+I_{2}+I_{3}=0.01 \mathrm{~cm}^{-1}$ which is rather small. For pure sulphur the only non zero contribution is $I_{2}$ estimated to be $1.6 \times 10^{-4} \mathrm{~cm}^{-1}$ Experimentally we got a $q$ independent temperature independent contribution of less than $2 \times 10^{-3} \mathrm{~cm}^{-1}$

We shall next discuss theoretical predictions about the correlation function $S(q)$.

\section{Theory.}

4.1 Determination of $S(q \rightarrow 0)=S(0)$. - Some years ago, the limit $n \rightarrow 0$ of a dilute $n$ vector model of magnetism in a small external field was introduced to describe equilibrium polymerization in a solvent with applications to sulphur solutions [13]. In the mean field approximation, this corresponds to the Flory theory of polymer solutions [17]. We have extended the theory to the study of the concentration correlation function $S(q)$ [18]. We first consider the limit $q \rightarrow 0$ where $S(q)$ tends to the concentration susceptibility $S(0)=\frac{\partial X_{s}}{\partial \tilde{\Delta}}$ From equation (5.15) and (5.19) of Wheeler and Pfeuty [13] and using the same notations, we get

$$
S(0)^{-1}=\left(\frac{\partial X_{\mathrm{s}}}{\partial \tilde{\Delta}}\right)^{-1}=-q \tilde{K}+\frac{1}{1-X_{\mathrm{s}}}+\frac{h}{m} \frac{[q \tilde{J} m+h]}{\left(h+\left(q \tilde{J} m+\frac{h}{2}\right)(q J m+h)^{2}\right)}
$$

where

$$
q \tilde{J}=\exp \left(\frac{\Delta H_{\mathrm{p}}}{T_{\mathrm{p}}^{*}}\left(1-\frac{T_{\mathrm{p}}^{*}}{T}\right)\right)
$$

with $\Delta H_{\mathrm{p}}=3.17 \mathrm{kcal} / \mathrm{mole}$ being the (positive) enthalpy of chain propagation and $T_{\mathrm{p}}^{*}=432 \mathrm{~K}$ being the polymerization transition of pure sulphur, so that

$$
q \tilde{J}=\exp \left(3.7\left(1-\frac{432}{T(\mathrm{~K})}\right)\right)
$$

$\tilde{\Delta}$ is the chemical potential of the solvent and $q \tilde{K}=\frac{4 T_{1}}{T}$ is a measure of solvent-sulphur interaction with $T_{1}$ being the upper critical solution point of the solvent sulphur solution. The « magnetic » field $\mathrm{h}$ is directly related to the enthalpy $\Delta H_{1}$ and entropy $\Delta S_{1}$ needed for the opening of a sulphur ring $S_{8}$.

$$
\frac{1}{2} h^{2}=K_{1}=\exp \left(\frac{T \Delta S_{1}-\Delta H_{1}}{k T}\right) .
$$


The « magnetic » order parameter $m$ is related to $h, X_{\mathrm{s}}$ and $q \tilde{J}$ through the additional equation (from Eqs. (5.14) and (5.15) of [13])

$$
X_{s}[q \tilde{J} m+h]=m\left[1+\frac{1}{2}(q \tilde{J} m+h)^{2}\right] .
$$

For given parameters $X_{s}, T, \Delta S_{1}, \Delta H_{1}, T_{1}$, from equations (4.3), (4.4) and (4.5) we get $m$ that we report in (4.1) to obtain $S(0)$. In the limit of $h$ going to zero $S(0)$ becomes, as a function of temperature, a step function with a discontinuity at the polymerization transition $T_{\mathrm{p}}$ which satisfies

$$
q \tilde{J}\left(T_{\mathrm{p}}\right) X_{\mathrm{s}}=1
$$

For $T<T_{\mathrm{p}}$ when $h \rightarrow 0 \mathrm{~m}$ also goes to zero (« disordered» phase) so that $S(0)^{-1}=$ $-\frac{4 T_{1}}{T}+\frac{1}{1-X_{s}}+\frac{1}{X_{s}}$ recovering the mean field behavior for a binary mixture [16]

$$
S(0)=\frac{X_{\mathrm{s}}\left(1-X_{\mathrm{s}}\right)}{1-\frac{4 T_{1}}{T} X_{\mathrm{s}}\left(1-X_{\mathrm{s}}\right)}
$$

Above $T_{\mathrm{p}}, m \neq 0$ when $h \rightarrow 0$ (ordered phase). The sulphur is polymerized, $S(0)$ is higher and given by

$$
\begin{aligned}
S(0)^{-1} & =-\frac{4 T_{1}}{T}+\frac{1}{1-X_{\mathrm{s}}} \\
S(0) & =\frac{\left(1-X_{\mathrm{s}}\right)}{1-\frac{4 T_{1}}{T}\left(1-X_{\mathrm{s}}\right)}
\end{aligned}
$$

In reality $h$ is not zero but small so that the discontinuity at $T_{\mathrm{p}}$ is rounded but still quite steep. From equation (4.8), $S(0)$ diverges when $\frac{4 T_{1}}{T}\left(1-X_{\mathrm{s}}\right)=1$ which, if combined with the polymerization transition $(h \rightarrow 0)$ given by equation (4.6), leads to the condition for a tricritical point at $T_{\mathrm{t}}, X_{\mathrm{s}}^{\mathrm{t}}$

$$
\begin{aligned}
\frac{4 T_{1}}{T_{\mathrm{t}}} & =\left(1-\left[q \tilde{J}\left(T_{\mathrm{t}}\right)\right]^{-1}\right)^{-1} \\
X_{\mathrm{t}} & =\left(q \tilde{J}\left(T_{\mathrm{t}}\right)\right)^{-1}
\end{aligned}
$$

A calculation of $S(0)$ as function of temperature is shown in figure 7 with $h=0.01$ $\left(T_{1} / T_{\mathrm{p}}^{*}\right)=0.65$ and with four values of $X_{\mathrm{s}}$ and in figure 8 with $h=0.01\left(T_{\mathrm{l}} / T_{\mathrm{p}}^{*}\right)=1.12$ and with four other values of $X_{\mathrm{s}}$. In figure 8 curve 4 corresponds to a «tricritical » like situation with $S(0)$ becoming very large.

4.2 Determination of $S(q)$. - Following the work of Furman and Blume [19] we have obtained for the dilute $n \rightarrow 0$ lattice model an Ornstein-Zernike expression of the concentration correlation function $S(q)=\frac{a+b q^{2}}{1+c q^{2}}$ where $a, b, c$ vary with temperature, and can be expressed as a function of $\left(q J, h, m, X_{5}\right)$ [18].

In figure 9 is shown the calculated correlation function $S(q)$ with $X_{\mathrm{s}}=0.6, h=0.01$, $\left(T_{1} / T_{\mathrm{p}}^{*}\right)=0.7$ at five different temperatures across the polymerization transition. 


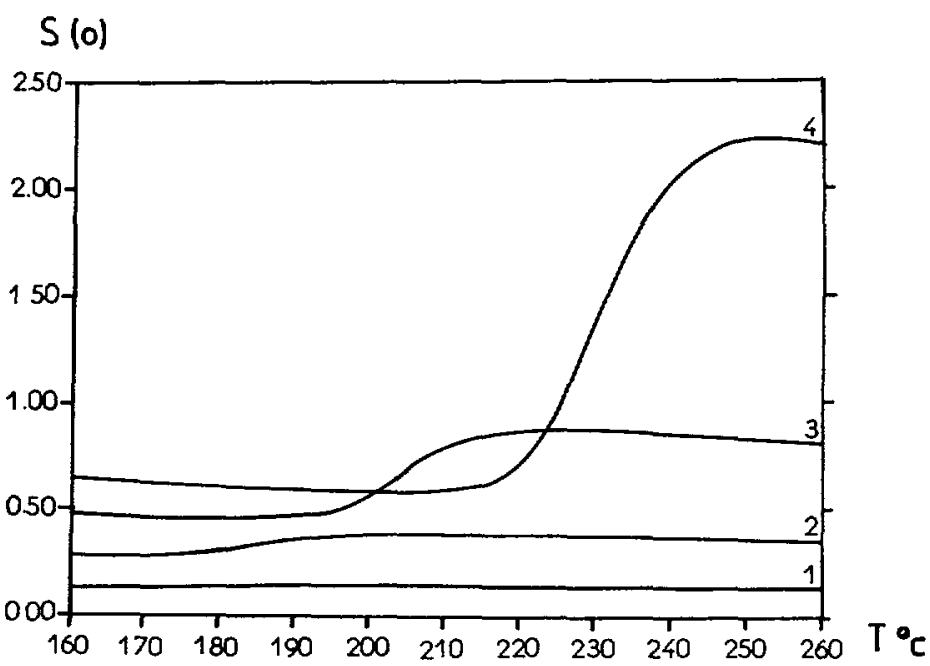

Fig. 7. - Theoretical calculation of $S(0)$ in absolute units as a function of temperature for parameters corresponding to sulphur + naphtalene $\mathrm{d}$. We choose $h=0.01$ and $\left(T_{1} / T_{\mathrm{p}}^{*}\right)=0.65$. The curves $1,2,3$ and 4 correspond respectively to $X_{\mathrm{s}}=0.9,0.8,0.7,0.6$.

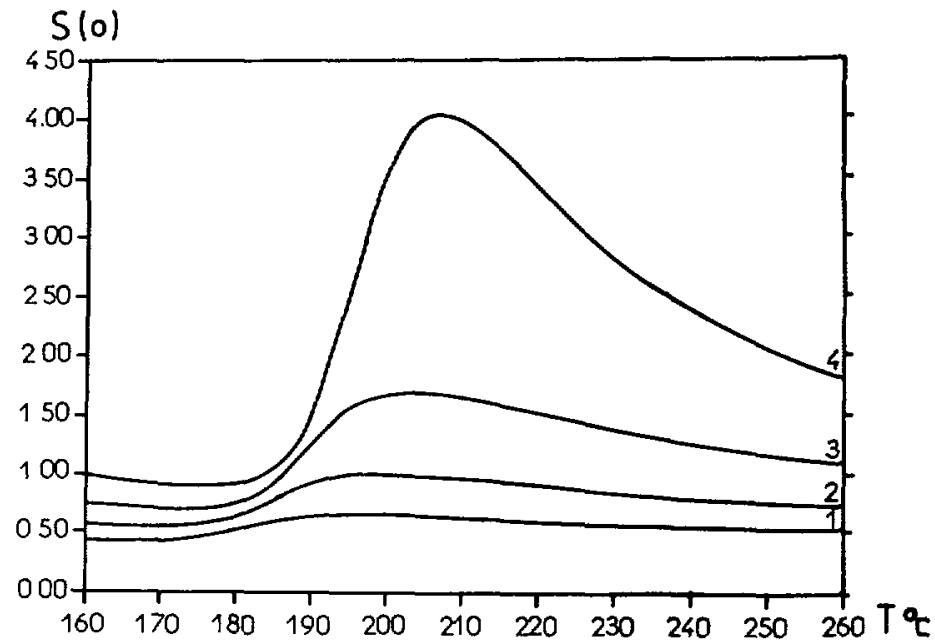

Fig. 8. - Theoretical calculation of $S(0)$ in absolute units as a function of temperature for parameters corresponding to sulphux + biphenyl d. We choose $h=0.01$ and $\left(T_{1} / T_{\mathrm{p}}^{*}\right)=1.12$. The curves $1,2,3$ and 4 correspond respectively to $X_{\mathrm{s}}=0.82,0.80,0.78,0.76$.

4.3 COMPARISON BETWEEN THEORY AND EXPERIMENTS. - For sulphur + naphtalene there is a qualitative agreement between experiments (curves 1 and 2 of Fig. 5) and theoretical predictions (curves 3 and 4 of Fig. 7). The sulphur-solvent interaction parameter $\left(T_{1} / T_{\mathrm{p}}^{*}\right)$ is chosen to be 0.65 (the best agreement is obtained with $\left.\left(T_{1} / T_{\mathrm{p}}^{*}\right)=0.7\right)$. This parameter is small so that no phase separation occurs at high temperature. For $X_{\mathrm{s}}$ small $\left(X_{\mathrm{s}}=0.6\right.$, $X_{\mathrm{s}}=0.55$ ) we were limited by the reactivity of the solvent with the chain ends at high temperature so that the runs had to be short $(10 \mathrm{~min})$. In the case of sulphur + biphenyl there is also a reasonable qualitative agreement between the experiments (curves 1 and 2 of Fig. 6) 


\section{$S(0)$}

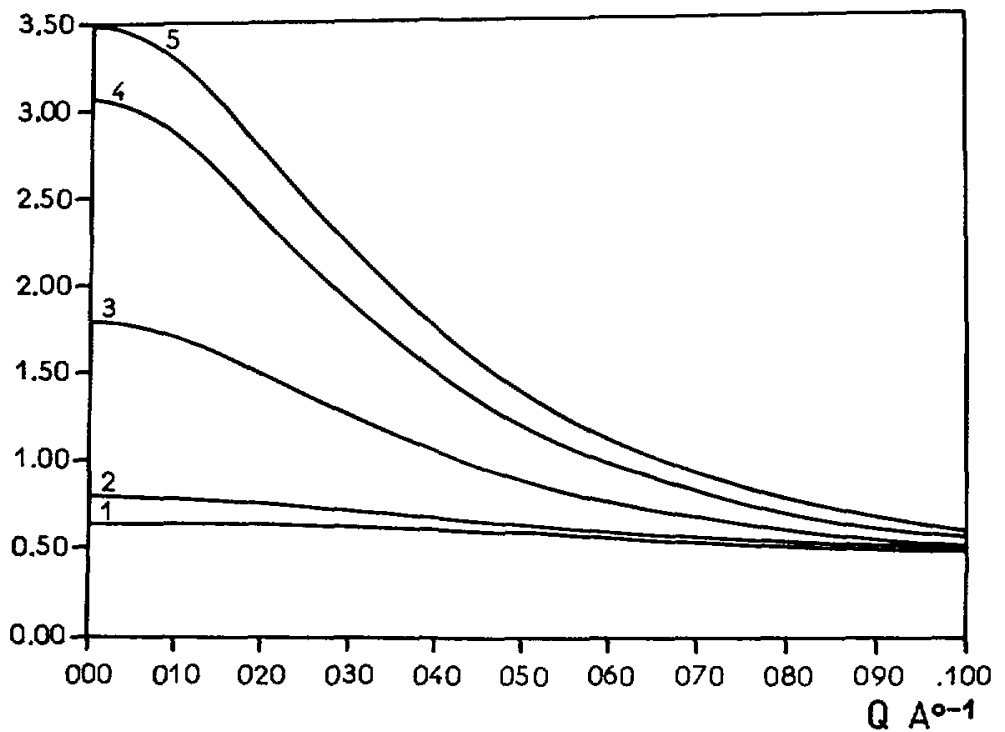

Fig. 9. - Theoretical calculation of $S(q)$ in absolute units as a function of $q$ in $\AA^{-1}$ for parameters corresponding to sulphur + naphtalene d. We choose $X_{s}=0.6, h=0.01$ and $\left(T_{1} / T_{\mathrm{p}}^{*}\right)=0.7$. The curves $1,2,3,4$ and 5 correspond respectively to the temperatures $T=210{ }^{\circ} \mathrm{C}, 220^{\circ} \mathrm{C}, 230{ }^{\circ} \mathrm{C}, 240^{\circ} \mathrm{C}$ and $250{ }^{\circ} \mathrm{C}$.

and the theoretical predictions (curves 3 and 4 of Fig. 8). In that case the chosen parameter $\left(T_{1} / T_{\mathrm{p}}^{*}\right)=1.12$ is higher than for naphtalene and a phase separation is expected at high temperature. In curve 2 of figure 6 , we approach this phase separation and $I(0)$ diverges when $T$ goes to $T_{\mathrm{c}}=225^{\circ} \mathrm{C}$ like $\left(T_{\mathrm{c}}-T\right)^{-1}$ This can be associated with tricritical like fluctuations. The irreversibility effects seen with naphtalene at high temperature, were not found with biphenyl which is known to be much less reactive. To do a better comparison with theory we should relate $I(0)$ to $S\left(q_{m}=0.9 \times 10^{-2} \AA^{-1}\right)$ using equation (3.5). The theory presented in 4.1 is a mean field theory which neglects critical effects. In fact for a true transition $(h \rightarrow 0)$ a weak «specific heat» like singularity is expected for $S(0)$ when $T$ approaches the polymerization transition $T_{\mathrm{p}}$ as predicted on general thermodynamic principles by Griffiths and Wheeler [20]. For $h \neq 0$ the rounding effects may suppress the weak singularity which has not been observed experimentally here. For $\mathrm{He}_{3}-\mathrm{He}_{4}$ solutions where a true superfluid 2 nd order phase transition takes place, such a weak singularity has also not been observed [14] but could be due there to the very small value of the specific heat exponent $\alpha$.

For the scattering function $S(q)$ there is also a qualitative agreement between experiments (Fig. 3) and theoretical calculation (Fig. 9). The theory presented in 4.2 is Ornstein Zernike like and cannot describe correctly the large $q$ behavior of the scattering function. For a conventional semi-dilute polymer solution this large $q$ part should vary as $q^{-1 / \nu}$ with $\frac{1}{\nu}=\frac{5}{3}$. In the present system $S(q)$ contains three contributions $S_{11}(q), S_{12}(q)$ and $S_{22}(q)$ (where index 1 is for sulphur in $S_{8}$ rings and 2 for sulphur in chains) and the quantity $S_{22}(q)$ for which conventional polymer scaling analysis could be applied [21] cannot be extracted from the measured total contribution $S(q)$. In the future, a non classical theory 
should be developed to calculate both $S(q)$ and the separate parts $S_{11}, S_{22}$ and $S_{12}$.

\section{Conclusion.}

For the first time, the polymerization of sulphur has been observed by SANS on a length scale of 10 to $100 \AA$ in the presence of a solvent for which concentration fluctuations are strongly induced by the polymerization transition and can be, for a deuterated solvent, clearly detected by neutron scattering. A simple lattice theory treated in the mean field approximation can explain the experimental measurements. We should also point the analogy between the present neutron scattering study of sulphur solutions and a previous light scattering study of $\mathrm{He}_{3}-\mathrm{He}_{4}$ solutions [15]. The present static experiment does not separate sulphur in chains from sulphur in $S_{8}$ rings. Hopefully this can be achieved by studying the slow dynamics as done recently by neutron spin echo techniques [22].

\section{Acknowledgements.}

We would like to thank J. P. Cotton, M.-C. Bellissent-Funel, A. Brulet and P. Chieux for discussions.

\section{References}

[1] MeYER B., Chem. Rev. 76 (1976) 367.

[2] BACON R. F. and FANELli, J. Am. Chem. Soc. 65 (1943) 539.

[3] Berthelot M., Ann. Ch. Phys. 49 (1857) 476.

[4] Tobolsky A. V. and Eisenberg A., J. Amer. Chem. Soc. 82 (1960) 289.

[5] Wheeler J. C., Kennedy S. J. and Pfeuty P., Phys. Rev. Lett. 45 (1980) 1748.

[6] DE Gennes P. G., Phys. Lett. 38 A (1972) 339.

[7] DE Gennes P. G., Scaling Concepts in Polymer Physics (Cornell University Press, Ithaca, N. Y., 1979).

[8] Bellissent R., Descotes L., Boue F. and Pfeuty P., Phys. Rev. B 41 (1990) 2135.

[9] Kennedy S. J. and Wheeler J. C., J. Chem. Phys. 78 (1983) 1523.

[10] LARkin J. A., KatZ J. and ScotT R. L., J. Phys. Chem. 71 (1967) 352.

[11] Anderson E. M. and Greer S. C., J. Chem. Phys. 88 (1988) 2666.

[12] SCOTT R. L., J. Chem. Phys. 69 (1965) 261.

[13] Wheeler J. C. and Pfeuty P., J. Chem. Phys. 74 (1981) 6415.

[14] Goellner G., Behringer R. and Meyer H., J. Low. Temp. Phys. 13 (1973) 113.

[15] Leiderer P., Watts D. R. and WebB W. W., Phys. Rev. Lett. 33 (1974) 483.

[16] Bhatia A. B. and Thornton D. E., Phys. Rev. B 2 (1970) 3004.

[17] Flory P., J. Chem. Phys. 10 (1942) 51.

[18] PFEUTY P., unpublished.

[19] Furman D. and Blume M., Phys. Rev. B 10 (1974) 2068.

[20] Griffiths R. B. and Wheeler J. C., Phys. Rev. A 2 (1970) 1047.

[21] Des Cloizeaux J. and Jannink G., Les Polymères en Solution (Editions de Physique, 1988).

[22] Pfeuty P., Boue F., Farago B. and Bellissent R., unpublished. 